\title{
Dual-Polarization Frequency Reuse with Frequency Band Shifting Allocation
}

\author{
Jingshown Wu, Senior Member, IEEE, and Meng-Che Wu
}

\begin{abstract}
Spectrum is a limited and precious resource in wireless communications. Effective spectrum utilization becomes more and more important because of the expeditious increase of demand for wireless communications. In principle, the dual-polarization frequency reuse system, which employs two orthogonally polarized electromagnetic waves to carry information, can double the system capacity. However, the system performance degrades seriously and sometimes becomes unacceptable due to cross polarization and fading. In this paper, a new linear-polarized dual-polarization frequency reuse system that utilizes the concept of orthogonal frequency allocation to increase spectrum utilization is proposed. We analyze the performance of the dual-polarization system modulated by the quadrature phase-shift keying in the multipath fading channel with cross-polarization interference and Doppler effect. Because of the orthogonality of the polarized carriers and frequency spacing, the effect of cross polarization, which defeats the performance of the dual-polarization system severely, can be ignored. The proposed system has a bit error rate around $10^{-7}$ at the bit energy-to-noise ratio of $14 \mathrm{~dB}$, while the bit error rates of the dual-polarization cancellation with bootstrap system and the conventional orthogonal frequency-division multiplexing system are about $10^{-4}$.
\end{abstract}

Index Terms-Dual-polarization, orthogonal frequency spacing.

\section{INTRODUCTION}

$\mathbf{I}$ $\mathrm{N}$ WIRELESS communications, spectrum is one of the most important resources. To increase spectrum utilization efficiency, many schemes, such as orthogonal frequency-division multiplexing (OFDM) [1]-[3], cellular frequency reuse, and dual-polarization frequency reuse [4], [5], were proposed and deployed. OFDM is an attractive technique for digital transmission. Spectrum utilization efficiency can be increased by the orthogonal frequency spacing and signal bands' overlapping. But the effects of frequency offset, multipath fading, and delay deeply affect the effectiveness of OFDM. It is impractical to use the OFDM scheme in wireless transmission without any modification and improvement. And the dual-polarization frequency reuse system, which uses orthogonally polarized electromagnetic (EM) waves, has been demonstrated in satellite communications. The linear and circular types of polarization are the most frequently used.

There are two directions of polarization (DoPs), vertical and horizontal, in the linear-polarized system, and two DoPs, clockwise and counterclockwise, in the circular-polarized system. In principle, transmission through orthogonal polarization car-

Manuscript received July 18, 1999; revised March 27, 2000. This work was supported by the Ministry of Education and the National Science Council, R.O.C., under Grants 89-E-FR-06-2-4 and NSC 88-2213-E-002-081.

The authors are with the Department of Electrical Engineering and Graduate Institute of Communication Engineering, National Taiwan University, Taipei, Taiwan 10617 R.O.C.

Publisher Item Identifier S 0018-9545(00)09172-6. riers doubles the system capacity. But practically, that is hard to achieve because of propagation impairments and antenna imperfections. The propagation impairments, such as rainfall attenuation, depolarization, and cross-polarization interference (CPI) deteriorate the signal transmission in the satellite-earth station links. Some compensation methods were reported in the literature [6]-[9]. Furthermore, the problems will become more complicated by the multipath fading effect when transmitting in the terrestrial environment.

Although some schemes, such as equalization and diversity, are proposed to overcome the problems, they are too complicated to be practical. In this paper, we adopt the concept of orthogonal frequency spacing used in OFDM and apply to the linear dual-polarization system. The signal band allocation and orthogonal frequency spacing between the vertical and horizontal DoPs are introduced. Therefore, the performance degradation problem can be easily solved. The proposed system may employ pilot symbols for channel coefficients estimation, carrier recovery, etc. Furthermore, band-edged pilot tones can be added in the proposed system, which is impossible for a conventional OFDM system. These issues will be discussed later.

The remainder of this paper is organized as follows. The system architecture and channel model are described in Section II. In Section III, the performance analysis is presented. We show that the performance of this system is much better than that of OFDM, even without using any complicated channel imperfection compensation mechanism. In Section IV, the influence of various factors on the system performance is discussed. Section V gives the conclusion.

\section{System ARchitecture AND ChanNel Models}

In the traditional dual-polarization frequency reuse system, the signal spectra of both DoPs completely overlap. Guard bands between signal spectra are needed to prevent adjacent channel interference due to the imperfection of bandpass filter. The difference between the proposed system and the traditional system is that the vertical polarization (i.e., $y$ DoP) signal spectra are located at the higher frequency band relative to the horizontal polarization (i.e., $x$ DoP) signal spectra by the amount of symbol rate $B$ in the proposed system. The system architecture and behavior are described as next.

\section{A. System Architecture}

The schematic of the transmitter is shown in Fig. 1. The function of the serial-to-parallel converter (SPC) will be described later, and the system description begins with the two input data streams $e_{i x}(t)$ and $e_{i y}(t)$, which are modulated by 


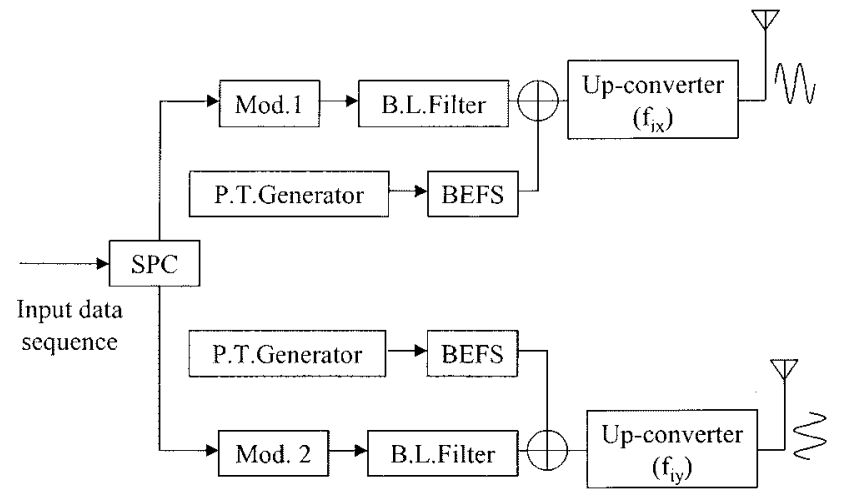

Fig. 1. The transmitter of the proposed system.

Modulator 1 (Mod.1) and Modulator 2 (Mod.2), respectively. Any modulation scheme can be adopted. In this paper, the dual quadrature phase-shift keying (QPSK) scheme is considered. After modulation, the modulated symbols are fed into the square-rooted raised cosine band-limited (BL) filters and then transmitted through the $x \mathrm{DoP}$ and $y$ DoP antennas. To maintain orthogonality and to estimate channel parameters, the narrow-band pilot tones (PTs), which are produced by the PT generators with the band-edged frequency shifter (BEFS), are added. The spectra allocation of PTs are chosen at the edges of the spectra of the corresponding in-band signals, as shown in Fig. 2(a). The number of pilot tones and their spacing interval depend on the channel complexity. Moreover, a pilot signal can be designed to carry more information about the channel in order to get better channel estimation. After adding the filtered symbols and pilot tones, the system up-converts the $x$ DoP signal and the $y$ DoP signal to two frequency bands $f_{i x}$ and $f_{i y}$, respectively, where $i$ denotes the $i$ th signal channel. $f_{i x}$ and $f_{i y}$ differ by the amount of symbol rate, so the two signals are linearly independent theoretically. If the width of the guard band is less than the signal bandwidth, the spectra of $x$ DoP signal in the $i$ th channel and $y$ DoP signal in the $(i-1)$ th channel overlap because of the band allocation. The sketch of signal spectrum allocation is depicted in Fig. 2(b), which shows that the two different DoP signal spectra in adjacent channels are separated by the value of bit rate plus $f_{d} . f_{d}$ can be considered as the width of guard band. Theoretically, if $f_{d}$ equals zero, the two signals are orthogonally spaced with zero interference. However, the value of $f_{d}$ can be adjusted to control the frequency band allocation for the spectra of PTs. On the other hand, $f_{d}$ is also prepared for compensating the Doppler effect. There is a limit that $f_{d}$ cannot be negative, i.e., overlapping the signal bands of the two adjacent channels in the same DoP is not allowed.

The schematic of the receiver is shown in Fig. 3. After downconverting the received signals, PT filtering and signal filtering are processed, respectively. The pilot tones are filtered out by the PT filters, and the signals are sent into the polarization recovery circuit and carrier recovery circuit, which we will not discuss further. The received symbols are filtered by the square-rooted raised cosine band-limited filters. The merit of using the filter is that we can take advantage of the properties of raised cosine filter without using other types of low-pass filters. Then the filtered symbols are fed into the demodulators with the reference recovered from the pilot tones. The detected data streams $\hat{e}_{i x}(t)$ and $\hat{e}_{i y}(t)$ are obtained. The function of the parallel-to-serial converter (PSC) will be described later.

\section{B. Channel Model}

In this paper, the multipath Rayleigh-fading channel with Doppler effect [10], [11] is considered. Taking the inherent limitation of dual-polarization transmission into account, we only analyze the two-ray case. The impulse responses of the dual polarized channel are given by

$$
h_{x}(t)=\alpha_{x} \delta(t)+\gamma_{x} A_{x} \delta\left(t-\tau_{x}\right) e^{j \varphi_{x}} e^{j 2 \pi f_{D} t}, \quad \text { for } x \operatorname{DoP}(1)
$$
and

$$
h_{y}(t)=\alpha_{y} \delta(t)+\gamma_{y} A_{y} \delta\left(t-\tau_{y}\right) e^{j \varphi_{y}} e^{j 2 \pi f_{D} t}, \quad \text { for } y \operatorname{DoP}(2)
$$

where

$\alpha_{j} \quad$ attenuation coefficient;

$\delta($.$) delta function;$

$f_{D}$ Doppler shift;

$\tau_{j} \quad$ delay time of the second path relative to the line-ofsight;

$\gamma_{j} \quad$ square-rooted power ratio with respect to the first path;

$\varphi_{j} \quad$ initial phase of the second path;

$A_{j} \quad$ Rayleigh random variable with unity second moment;

$j=\left\{\begin{array}{ll}x, & x \mathrm{DoP} \\ y, & y \mathrm{DoP}\end{array}\right.$.

Because Doppler effect results from the relative velocity between the transmitter and receiver, it is reasonable to set the same Doppler shift $f_{D}$ in both $x$ DoP and $y$ DoP.

Pilot tones are conducive to the estimation of the channel parameters. There is at least one pilot tone in each coherent bandwidth. The theoretical functions of reflection coefficients of horizontal-polarized wave and vertical-polarized wave were discussed in the literature [10]. For horizontal polarization, the function indicates that the relative phase of the incident and reflected waves is nearly $180^{\circ}$ for all angles of incidence. But for vertical polarization, the results are quite different. When the angle of incidence is small, the behaviors of horizontal and vertical polarization are almost the same. But as the angle of incidence increases, the magnitude and relative phase of the reflected wave decrease rapidly. It is a torment to recover the vertical polarization wave. However, with the existence of pilot tones in the system, this problem can be solved. Both reflection coefficients are frequency dependent. Therefore, the relative phase changes of both pilot tone and in-band signal are almost the same. We can recover the carrier phase by the pilot tones easily.

\section{Data Flow Issue}

In the traditional dual-polarized systems, each DoP is used as an independent transmission channel. It means that the data streams transmitted in both DoPs are uncorrelated. For better transmission performance, the error correction code (e.g., convolutional code) can be used by expanding the code word by some redundant bits. The training sequence, which estimates the channel characteristic, can also be used. To save the transmission payload, the concept of parallel transmission in OFDM [1] can be introduced to reduce the code redundancy. In Fig. 1, the 


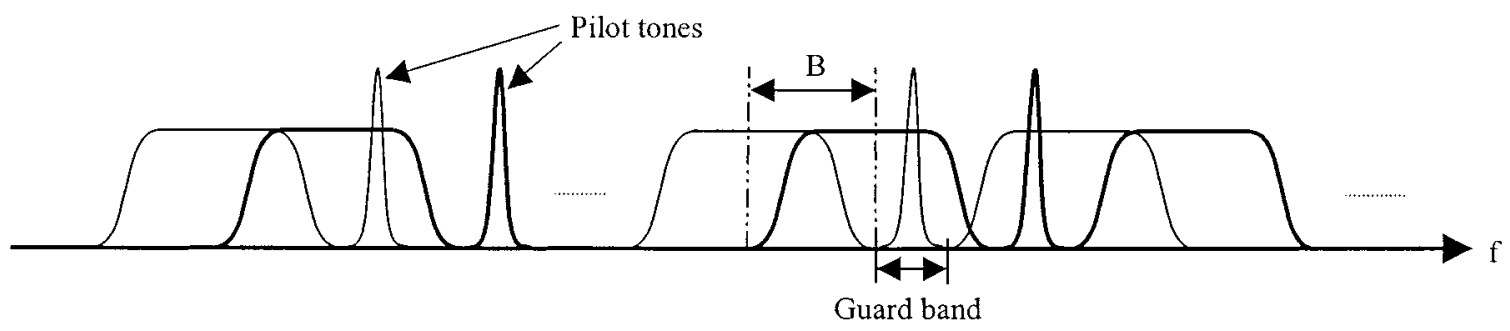

(a) The overall signal spectrum include pilot tones.
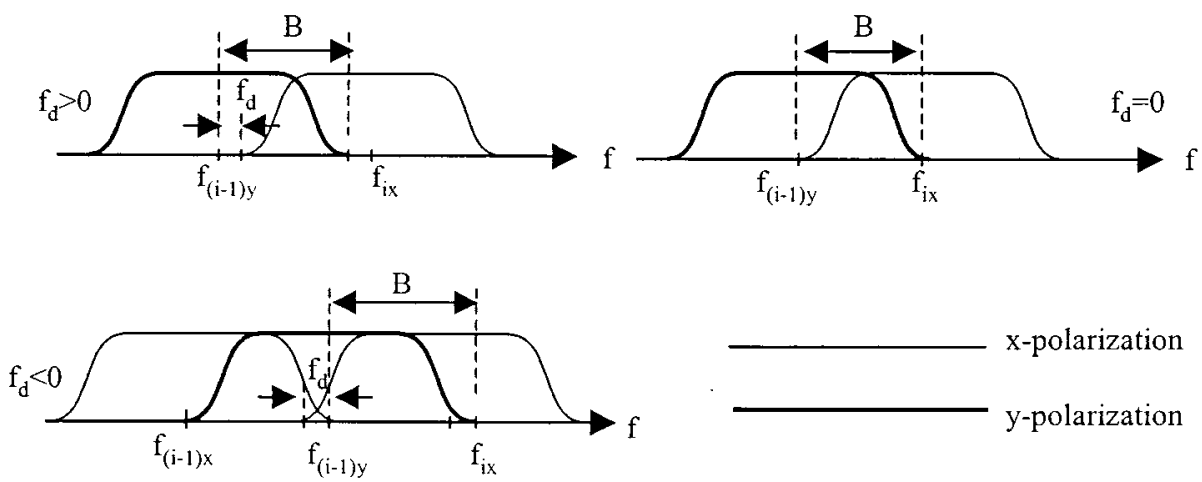

(b) Diagram of CPI due to frequency overlap.

Fig. 2. Sketch of frequency band allocation and frequency shifting.

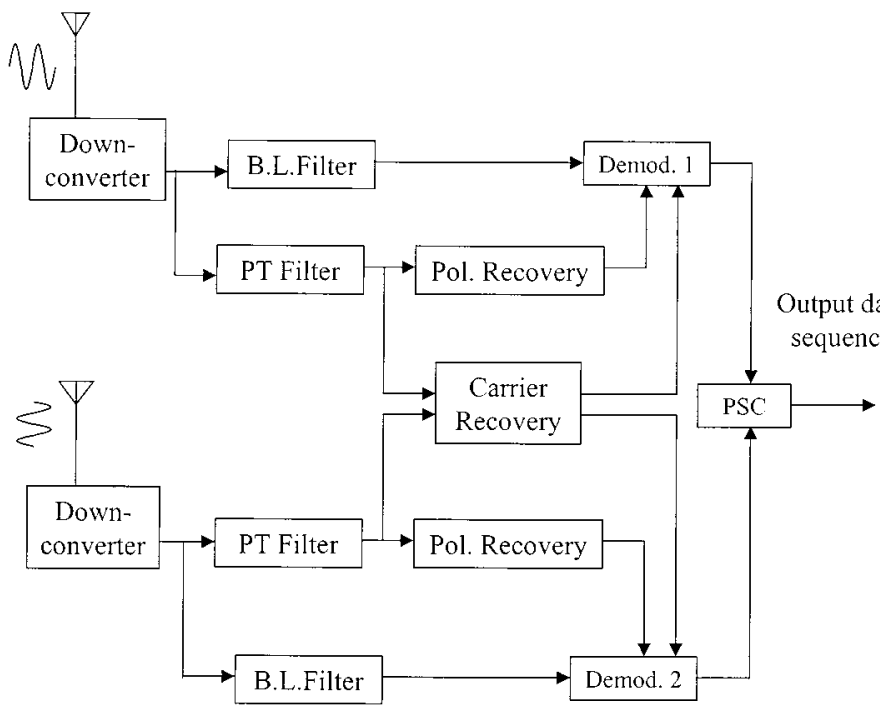

Fig. 3. The receiver of the proposed system.

coded data stream is fed into the SPC and split into two data sequences $e_{i x}(t)$ and $e_{i y}(t)$. The consequent processes were indicated in the previous sections. The receiving processes in the receiver are also illustrated in Fig. 3. Finally, the desired coded data stream is obtained by sending the detected data sequences $\hat{e}_{i x}(t)$ and $\hat{e}_{i y}(t)$ through the PSC.

\section{SySTEM ANALYSIS}

Here we consider the dual-QPSK system and express the input data sequence to the signal space in complex domain. The symbols of $x$ and $y$ DoP in the $k$ th duration are denoted by

$$
\begin{aligned}
& u_{i x}(t-k T)=a_{i}(t-k T)+j b_{i}(t-k T) \\
& u_{i y}(t-k T)=c_{i}(t-k T)+j d_{i}(t-k T)
\end{aligned}
$$

where $a_{i}(t)$ and $c_{i}(t)$ are the QPSK signals in the I-branch of $x$ and $y$ DoPs in the $i$ th channel, respectively. $b_{i}(t)$ and $d_{i}(t)$ are the QPSK signals in the Q-branch of both DoPs in the $i$ th channel. Thereafter, $a_{k}^{i}$ will represent $a_{i}(t-k T)$ for convenience. $T$ denotes the symbol duration of the QPSK modulators. The outputs of the modulators are sampled every $T$ seconds and then passed through the square-root raised cosine filter with the impulse response $p(t)$. After up converting the mapped signals to the carrier frequencies, we get the transmitting signals as

$$
\begin{aligned}
& S_{i x}(t)=\sum_{k=-\infty}^{\infty} \sqrt{2} u_{i x}(t-k T) p(t-k T) \exp \left(j 2 \pi f_{i x} t\right) \\
& S_{i y}(t)=\sum_{k=-\infty}^{\infty} \sqrt{2} u_{i y}(t-k T) p(t-k T) \exp \left(j 2 \pi f_{i y} t\right)
\end{aligned}
$$

Because of the orthogonality between $S_{i x}(t)$ and $S_{i y}(t)$, they do not interfere with each other. However, $S_{i x}(t)$ will be interfered by the adjacent channel signals $S_{(i-1) y}(t)$ and the delayed signal due to propagation imperfection. Similarly, $S_{i y}(t)$ will be interfered with by $S_{(i+1) x}(t)$ and the delayed signal. Here we only present the $x$ DoP case because the other is exactly the same. 
At the receiver, the received signal of $x \mathrm{DoP}$ is given by

$$
r_{i x}(t)=y_{i x}(t)+n_{i x}(t)
$$

where $n_{i x}(t)$ is the additive white Gaussian random process with zero mean and the variance $N_{0} / 2 . y_{i x}(t)$ is the $x$ DoP channel output with the inputs $S_{i x}(t)$ and $S_{(i-1) y}(t)$. It can be expressed as

$$
\begin{aligned}
y_{i x}(t)=\operatorname{Re}\{ & \alpha_{x}\left(S_{i x}(t)+R S_{(i-1) y}(t)\right) \\
& +\beta_{1}\left(\gamma_{x} A_{x} S_{i x}\left(t-\tau_{x}\right) e^{j \varphi_{x}}\right. \\
& \left.+R \gamma_{y} A_{y} S_{(i-1) y}\left(t-\tau_{y}\right) e^{j \varphi_{y}}\right) \\
& \left.\cdot e^{j 2 \pi f_{D} t}\right\}
\end{aligned}
$$

The first term is the line-of-sight signal. The second term is the delayed signal and interference with delay time $\tau_{x}$ and $\tau_{y}$ of $x$ and $y$ DoPs, respectively. $\alpha_{x}$ is the attenuation coefficient. Coefficient $R$ is the cross-polarization coefficient, satisfying $0 \leq R \leq 1$. $\beta_{1}$ is the polarization-mismatch coefficient of delayed symbol of the second path. It lies between zero and one. The frequency $f_{D}$ is the Doppler shift in the second path. In the following analysis, we will take values all normalized to $\alpha_{x}$, i.e., $\alpha_{x}=1$.

At the BL filter output, we get the sampled signal $Z_{i x}$, which can be expressed as

$$
\begin{aligned}
Z_{i x}(n T)= & \left.r_{i x}(t) \sqrt{2} \cos \left(2 \pi f_{i x} t\right) * p(t)\right|_{t=n T} \\
= & \sum_{k=-\infty}^{\infty} a_{k}^{i} p(t-k T) * p(t) \\
& +R \sum_{k=-\infty}^{\infty}\left(c_{k}^{i-1} p(t-k T)\right. \\
& \cdot \cos \left(2 \pi\left(f_{(i-1) y}-f_{i x}\right) t\right) \\
& -d_{k}^{i-1} p(t-k T) \\
& \left.\cdot \sin \left(2 \pi\left(f_{(i-1) y}-f_{i x}\right) t\right)\right) * p(t) \\
& +\beta_{1} \gamma_{x} A_{x} \cos \alpha_{1} \sum_{k=-\infty}^{\infty}\left(a_{k}^{i} p\left(t-k T-\tau_{x}\right)\right. \\
& \left.\cdot \cos 2 \pi f_{D} t-b_{k}^{i} p\left(t-k T-\tau_{x}\right) \sin 2 \pi f_{D} t\right) * p(t) \\
& +\beta_{1} \gamma_{x} A_{x} \sin \alpha_{1} \sum_{k=-\infty}^{\infty}\left(a_{k}^{i} p\left(t-k T-\tau_{x}\right)\right. \\
& \left.\cdot \sin 2 \pi f_{D} t+b_{k}^{i} p\left(t-k T-\tau_{x}\right) \cos 2 \pi f_{D} t\right) * p(t) \\
& +R \beta_{1} \gamma_{y} A_{y} \cos \alpha_{2} \sum_{k=-\infty}^{\infty}\left(c_{k}^{i-1} p\left(t-k T-\tau_{y}\right)\right. \\
& \cdot \cos \left(2 \pi\left(f_{(i-1) y}-f_{i x}+f_{D}\right) t\right) \\
& -d_{k}^{i-1} p\left(t-k T-\tau_{y}\right) \\
& \left.\cdot \sin \left(2 \pi\left(f_{(i-1) y}-f_{i x}+f_{D}\right) t\right)\right) * p(t) \\
& +R \beta_{1} \gamma_{y} A_{y} \sin \alpha_{2} \sum_{k=-\infty}^{\infty}\left(c_{k}^{i-1} p\left(t-k T-\tau_{y}\right)\right.
\end{aligned}
$$

$$
\begin{aligned}
& \cdot \sin \left(2 \pi\left(f_{(i-1) y}-f_{i x}+f_{D}\right) t\right) \\
& +d_{k}^{i-1} p\left(t-k T-\tau_{y}\right) \\
& \left.\cdot \cos \left(2 \pi\left(f_{(i-1) y}-f_{i x}+f_{D}\right) t\right)\right) * p(t)+N
\end{aligned}
$$

and

$$
N=\left.n_{i x}(t) \sqrt{2} \cos \left(2 \pi f_{i x} t\right) * p(t)\right|_{t=n T}
$$

where $\alpha_{1}=2 \pi f_{i x} \tau_{x}-\varphi_{x}$, and $\alpha_{2}=2 \pi f_{(i-1) y} \tau_{y}-\varphi_{y} . N$ is the filtered Gaussian noise with zero mean and the variance $N_{0} / 2$.

Let

$$
\begin{aligned}
& q_{1}(t-k T, x)=\left(p(t-k T) \cos \left(\frac{2 \pi x t}{T}\right)\right) * p(t) \\
& q_{2}(t-k T, x)=\left(p(t-k T) \sin \left(\frac{2 \pi x t}{T}\right)\right) * p(t) .
\end{aligned}
$$

After some manipulation, we get

$$
q_{1}(t-k T, x)=\cos \left(\frac{\pi x}{T}(t+k T)\right) m(t-k T, x)
$$

and

$$
q_{2}(t-k T, x)=\sin \left(\frac{\pi x}{T}(t+k T)\right) m(t-k T, x)
$$

where $m(t, x)$ is given in Table $\mathrm{I}$. The detailed derivation is presented in Appendix I.

In the following analysis, we consider the two kinds of channel characteristics.

\section{A. Mutually Dependent Rayleigh Fading Channel}

In this case, the amplitude coefficients of $x$ - and $y$-directions of polarization are dependent, i.e., $A_{x}$ is a function of $A_{y}$. For simplicity, we choose $A_{x}=A_{y}$, which indicates that the amplitude coefficients of two DoPs are positively correlated. At the output of the correlator in the receiver, we get

$$
\begin{aligned}
Z_{i x}(k=0) & =\left.\left(r_{i x}(t) \sqrt{2} \cos \left(2 \pi f_{i x} t\right)\right) * p(t)\right|_{t=0} \\
& =a_{0}+X_{1}+A X_{2}+N
\end{aligned}
$$

where $A=A_{x}=A_{y} \cdot X_{1}$ is cross-polarization interference of the current symbol. $X_{2}$ is the sum of the delayed-symbol interferences (DSIs) of the current and the last symbol corresponding to $x$ - and $y$-directions of polarization. $N$ is the low-pass additive white Gaussian noise (AWGN) with variance $N_{0} / 2$. By the assumption of $\tau_{x}<T$ and $\tau_{y}<T$, only the interferences from the current symbol and the last symbol have to be considered. According to (9), $X_{1}$ and $X_{2}$ can be expressed as

$$
\begin{aligned}
X_{1}=R & \left(c_{0}^{i-1} q_{1}(0, \delta+1)+c_{-1}^{i-1} q_{1}(-T, \delta+1)\right. \\
& \left.-d_{0}^{i-1} q_{2}(0, \delta+1)-d_{-1}^{i-1} q_{2}(-T, \delta+1)\right)
\end{aligned}
$$


TABLE I

Solution Functions of CONVOLUTION PROCESS With DifFERENT FreQUENCy BAND ALLOCATION

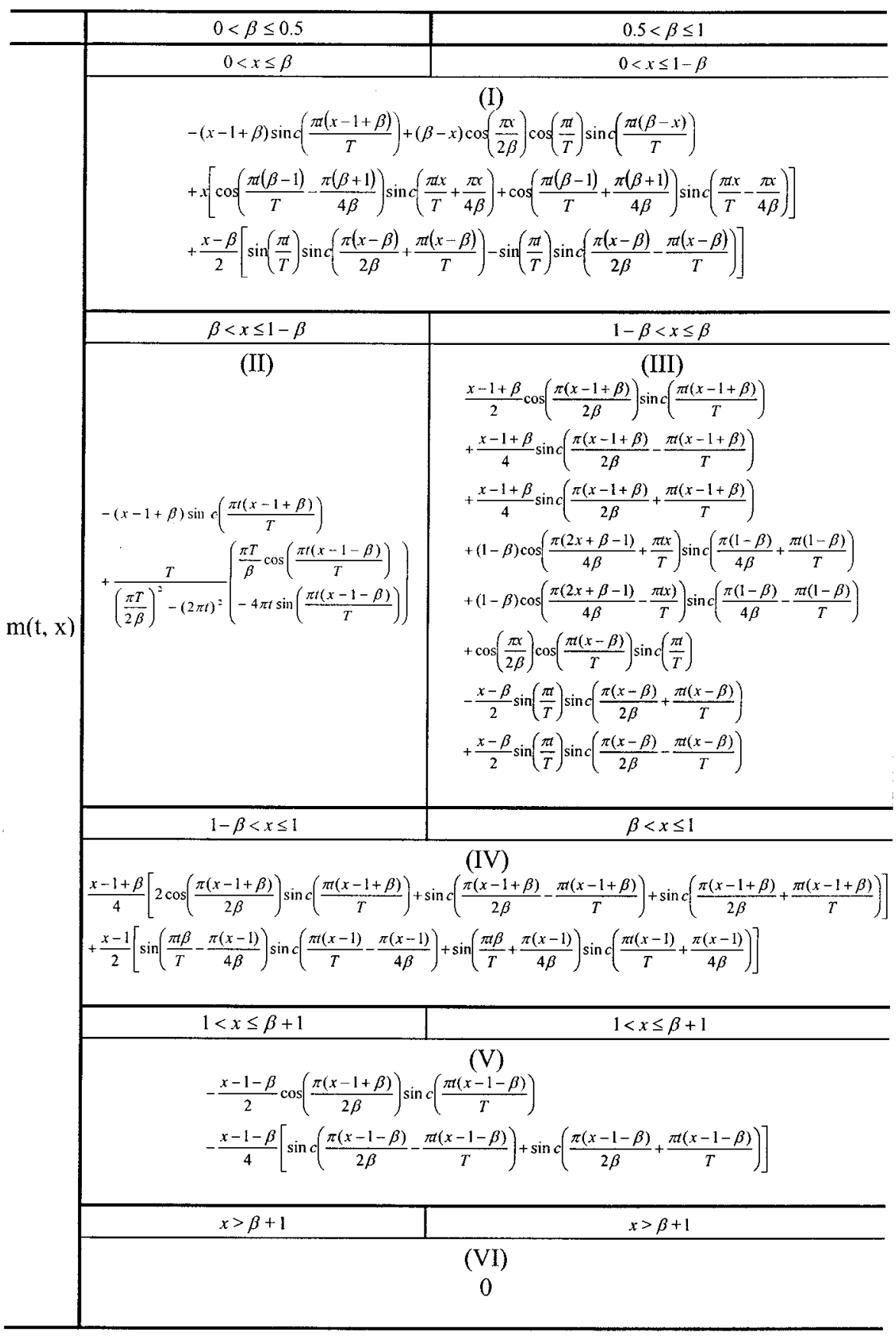

and

$$
\begin{aligned}
X_{2}=\beta_{1} \gamma\{ & \left(a_{0}^{i} \cos \alpha_{1}+b_{0}^{i} \sin \alpha_{1}\right) q_{1}\left(-\tau_{x}, \Delta\right) \\
& +\left(a_{-1}^{i} \cos \alpha_{1}+b_{-1}^{i} \sin \alpha_{1}\right) q_{1}\left(-T-\tau_{x}, \Delta\right) \\
& +\left(a_{0}^{i} \sin \alpha_{1}-b_{0}^{i} \cos \alpha_{1}\right) q_{2}\left(-\tau_{x}, \Delta\right) \\
& +\left(a_{-1}^{i} \sin \alpha_{1}-b_{-1}^{i} \cos \alpha_{1}\right) q_{2}\left(-T-\tau_{x}, \Delta\right) \\
& +R\left[\left(c_{0}^{i-1} \cos \alpha_{2}+d_{0}^{i-1} \sin \alpha_{2}\right)\right. \\
& \cdot q_{1}\left(-\tau_{y}, \delta+\Delta+1\right) \\
& +\left(c_{-1}^{i-1} \cos \alpha_{2}+d_{-1}^{i-1} \sin \alpha_{2}\right)
\end{aligned}
$$

$$
\begin{aligned}
& \cdot q_{1}\left(-T-\tau_{y}, \delta+\Delta+1\right) \\
& +\left(c_{0}^{i-1} \sin \alpha_{2}-d_{0}^{i-1} \cos \alpha_{2}\right) \\
& q_{2}\left(-\tau_{y}, \delta+\Delta+1\right) \\
& +\left(c_{-1}^{i-1} \sin \alpha_{2}-d_{-1}^{i-1} \cos \alpha_{2}\right) \\
& \left.\left.\cdot q_{2}\left(-T-\tau_{y}, \delta+\Delta+1\right)\right]\right\}
\end{aligned}
$$

where $a_{0}^{i}, b_{0}^{i}, c_{0}^{i-1}$, and $d_{0}^{i-1}$ are the bits of the current symbol. $a_{-1}^{i}, b_{-1}^{i}, c_{-1}^{i-1}$, and $d_{-1}^{i-1}$ are that of the last symbol. $\delta=f_{d} \cdot T$ is the normalized shifting frequency and $\Delta=f_{D} \cdot T$ is the normalized Doppler shift frequency. 
Then the bit error probability is given by

$$
\begin{aligned}
P e_{\text {dep }}= & \frac{1}{2}\left(\operatorname{Pr}\left\{Z_{i x}<0 \mid a_{0}^{i}=1\right\}+\operatorname{Pr}\left\{Z_{i x}>0 \mid a_{0}^{i}=-1\right\}\right) \\
= & \operatorname{Pr}\left\{Z_{i x}<0 \mid a_{0}^{i}=1\right\} \\
= & \left(\frac{1}{2 \pi}\right)^{2} \int_{-\pi}^{\pi} \int_{-\pi}^{\pi}\left(Q\left(\sqrt{2 z}\left(1+X_{1}\right)\right)\right. \\
& -x \sqrt{\frac{2 z}{1+2 z x^{2}}} \exp \left[-\frac{z\left(1+X_{1}\right)^{2}}{1+2 z x^{2}}\right] \\
& \left.\cdot Q\left(\frac{2 z x\left(1+X_{1}\right)}{\sqrt{1+2 z x^{2}}}\right)\right)_{x=X_{2}} d \alpha_{1} d \alpha_{2} .
\end{aligned}
$$

The detailed derivation is given in Appendix II.

\section{B. Independent Rayleigh Fading Channel}

In the most random case, we assume that $A_{x}$ and $A_{y}$ are independent and identically Rayleigh distributed with unity second moment. There may be different power ratios for each direction of polarization. For simplicity, we set the power ratios for both directions of polarization to the same value.

At the output of the correlator in the receiver, we get

$$
\begin{aligned}
Z_{i x}(k=0) & =\left.\left(r_{i x}(t) \sqrt{2} \cos \left(2 \pi f_{i x} t\right)\right) * p(t)\right|_{t=0} \\
& =a_{0}+Y_{1}+A_{x} Y_{2}+A_{y} Y_{3}+N
\end{aligned}
$$

where $Y_{1}$ is the cross-polarization interference of the current symbol. $Y_{2}$ is the sum of the DSI of the current and the last symbol at the $x$-direction of polarization. Similarly, $Y_{3}$ is the DSI at the $y$-direction polarization. $N$ is the low-pass AWGN with variance $N_{0} / 2$. Based on the assumption of $\tau_{x}<T$ and $\tau_{y}<T$, only the interference from the current symbol and the last symbol are considered. According to (9), $Y_{1}, Y_{2}$, and $Y_{3}$ can be expressed as follows:

$$
\begin{aligned}
Y_{1}=R( & c_{0}^{i-1} q_{1}(0, \delta+1)+c_{-1}^{i-1} q_{1}(-T, \delta+1) \\
& \left.-d_{0}^{i-1} q_{2}(0, \delta+1)-d_{-1}^{i-1} q_{2}(-T, \delta+1)\right) \\
Y_{2}=\beta_{1} \gamma[ & \left(a_{0}^{i} \cos \alpha_{1}+b_{0}^{i} \sin \alpha_{1}\right) q_{1}\left(-\tau_{x}, \Delta\right) \\
& +\left(a_{-1}^{i} \cos \alpha_{1}+b_{-1}^{i} \sin \alpha_{1}\right) q_{1}\left(-T-\tau_{x}, \Delta\right) \\
& +\left(a_{0}^{i} \sin \alpha_{1}-b_{0}^{i} \cos \alpha_{1}\right) q_{2}\left(-\tau_{x}, \Delta\right) \\
& \left.+\left(a_{-1}^{i} \sin \alpha_{1}-b_{-1}^{i} \cos \alpha_{1}\right) q_{2}\left(-T-\tau_{x}, \Delta\right)\right]
\end{aligned}
$$

$$
\begin{aligned}
Y_{3}=\beta_{1} \gamma R[( & \left(c_{0}^{i-1} \cos \alpha_{2}+d_{0}^{i-1} \sin \alpha_{2}\right) \\
& \cdot q_{1}\left(-\tau_{y}, \delta+\Delta+1\right) \\
& +\left(c_{-1}^{i-1} \cos \alpha_{2}+d_{-1}^{i-1} \sin \alpha_{2}\right) \\
& \cdot q_{1}\left(-T-\tau_{y}, \delta+\Delta+1\right) \\
& +\left(c_{0}^{i-1} \sin \alpha_{2}-d_{0}^{i-1} \cos \alpha_{2}\right) \\
& \cdot q_{2}\left(-\tau_{y}, \delta+\Delta+1\right) \\
& +\left(c_{-1}^{i-1} \sin \alpha_{2}-d_{-1}^{i-1} \cos \alpha_{2}\right) \\
& \left.\cdot q_{2}\left(-T-\tau_{y}, \delta+\Delta+1\right)\right]
\end{aligned}
$$

Now, we can compute the bit error probability of the system using dual-QPSK. The bit error rate (BER) is given by

$$
\begin{aligned}
& P e= \frac{1}{2}\left(\operatorname{Pr}\left\{Z_{i x}<0 \mid a_{0}^{i}=1\right\}\right. \\
&\left.+\operatorname{Pr}\left\{Z_{i x}>0 \mid a_{0}^{i}=-1\right\}\right) \\
&= \operatorname{Pr}\left\{Z_{i x}<0 \mid a_{0}^{i}=1\right\} \\
&= P e_{\operatorname{dep}}\left(Y_{3}\right)-\left(\frac{1}{2 \pi}\right)^{2} \int_{-\pi}^{\pi} \int_{-\pi}^{\pi} \\
& \cdot\left\{\frac{Y_{2} \sqrt{2 z} \sqrt{1+2 z Y_{2}^{2}}}{1+2 z Y_{2}^{2}+2 z Y_{3}^{2}}\right. \\
& \cdot\left[e^{-\left(\left(z\left(1+Y_{1}\right)^{2}\right) /\left(1+2 z Y_{2}^{2}\right)\right)}\right. \\
& \cdot Q\left(\frac{2 z Y_{2}\left(1+Y_{1}\right)}{\sqrt{1+2 z Y_{2}^{2}}}\right)-\frac{2 z Y_{2} Y_{3}}{\sqrt{\left(1+2 z Y_{2}^{2}\right)\left(1+2 z Y_{3}^{2}\right)}} \\
&\left.\cdot e^{-\left(\left(z\left(1+Y_{1}\right)^{2}\right) /\left(1+2 z Y_{3}^{2}\right)\right)} Q\left(\frac{2 z Y_{3}\left(1+Y_{1}\right)}{\sqrt{1+2 z Y_{3}^{2}}}\right)\right] \\
&\left.\cdot e^{-\left(\left(z\left(1+Y_{1}\right)^{2}\right) /\left(1+2 z Y_{2}^{2}+2 z Y_{3}^{2}\right)\right)} \cdot \operatorname{Res}\right\} d \alpha_{1} d \alpha_{2} \\
&\sqrt{\pi((2 z)-1})
\end{aligned}
$$

where Res is the residual part of the equation. It can be expressed as

$$
\operatorname{Re} s= \begin{cases}\int_{B_{1}}^{\infty} \int_{B_{2}}^{B_{3}} e^{-A_{y}^{2}} e^{-n^{2} / 2} d A_{y} d n, & Y_{2} Y_{3}>0 \\ & \int_{-\infty}^{B_{1}} \int_{B_{3}}^{\infty} e^{-A_{y}^{2}} e^{-n^{2} / 2} d A_{y} d n \\ & +\int_{B_{1}}^{\infty} \int_{B_{2}}^{\infty} e^{-A_{y}^{2}} e^{-n^{2} / 2} d A_{y} d n, \quad Y_{2} Y_{3} \leq 0\end{cases}
$$

and

$$
\begin{aligned}
& B_{1}=\frac{2 z Y_{2}\left(1+Y_{1}\right)}{\sqrt{1+2 z Y_{2}^{2}}} \\
& B_{2}=\frac{2 z Y_{3}\left(1+Y_{1}\right)}{\sqrt{\left(1+2 z Y_{2}^{2}+2 z Y_{3}^{2}\right)\left(2+4 z Y_{2}^{2}\right)}} \\
& B_{3}=\frac{1}{2 z Y_{2} Y_{3}} \sqrt{\frac{1+2 z Y_{2}^{2}+2 z Y_{3}^{2}}{2}}\left(n-B_{1}\right)+B_{2} .
\end{aligned}
$$

The detailed derivation is shown in Appendix III.

Now we take the intersymbol interference (ISI) effect into consideration. Because of different symbol combinations, there are different BER performances. We have to average the performance including the ISI effect over all cases given for the current and the last bits. By the assumption of equally probable bit combination, the averaged BER is

$$
\begin{aligned}
P e_{\text {ave }} & =\frac{1}{256} \sum_{a_{0}^{i}=-1}^{1} \sum_{b_{0}^{i}=-1}^{1} \ldots \sum_{d_{-1}^{i-1}=-1}^{1} \operatorname{Pe}\left(a_{0}^{i}, b_{0}^{i}, \ldots, d_{-1}^{i-1}\right) \\
& =\frac{\operatorname{Pe}\left(a_{0}^{i}, b_{0}^{i}, \ldots, d_{-1}^{i-1}\right) .}{}
\end{aligned}
$$




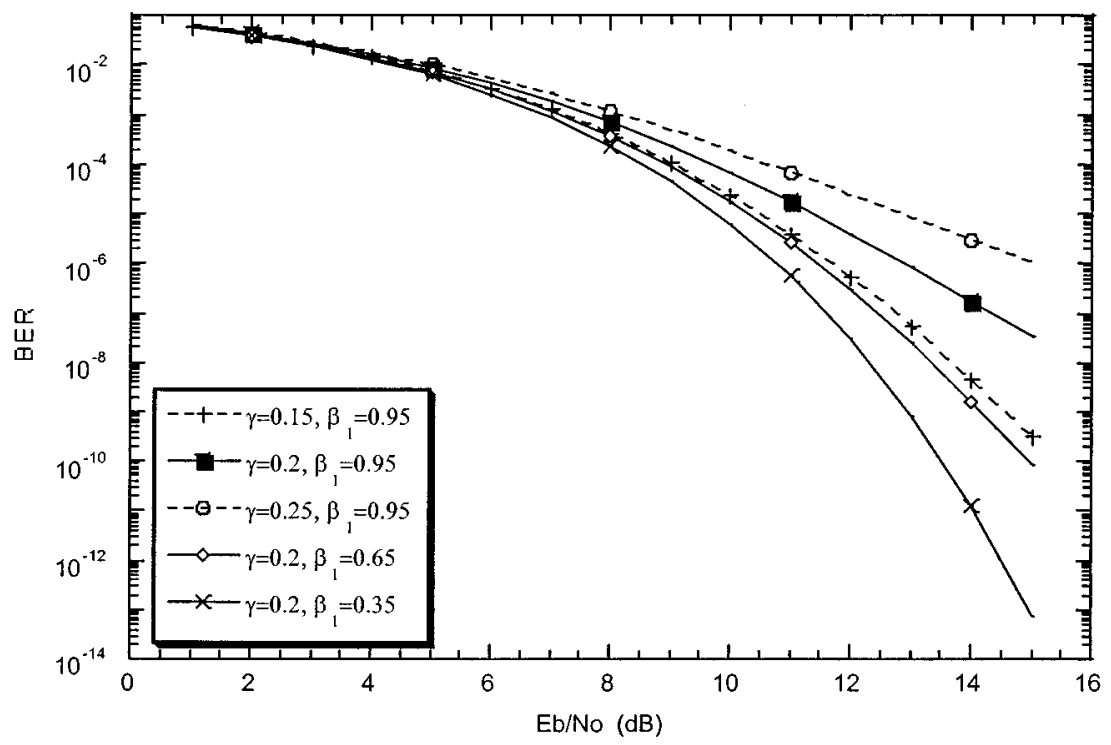

Fig. 4. Performance with various values of $\gamma$ and $\beta_{1}$ for $\Delta_{1}=0.2, \Delta_{2}=0.3, \delta=0.3, R=0.2 \beta=0, \Delta=0.2$, and $\beta_{1}=0.95$.

\section{DISCUSSION}

The BER performance is influenced by several factors. In this section, the performance affected by these factors is discussed and a comparison with other systems is made.

\section{A. Power Strength of the Second Path}

The influence of the square-root power ratio of the first and the second paths $\gamma$ and the correlation between the polarizations of line-of-sight and the second path $\beta_{1}$ is shown in Fig. 4. When $\gamma$ increases from 0.15 to 0.25 at $\beta_{1}=0.95$, the system performance is degraded about $2 \mathrm{~dB}$ at the BER of $10^{-4}$. For fixed $\gamma=0.2$, the performance degrades about $1.5 \mathrm{~dB}$ when $\beta_{1}$ increases from 0.35 to 0.95 . It implies that the performance is sensitive to the power strength of the second path signal. Because of the delay of the second path, the effect of ISI has to be considered. The BER expressed in (25) is the averaged performance of all bit-combination patterns in a symbol duration interval. In general, the averaged BER is dominant by the worst case. Therefore, the performance will be better with smaller $\beta_{1}$, i.e., the lower polarization correlation between the line-of-sight signal and the second-path signal yields better performance.

\section{B. Effect of Cross-Polarization Interference (CPI)}

The performance for various values of the CPI coefficient $R$ is shown in Fig. 5. It indicates that CPI does not affect the performance at all. The reason is that the orthogonal frequency shifting has eliminated the damage of cross-polarization interference. This is the merit of the system, and we can save the effort to compensate cross-polarization problems. The anti-CPI characteristic can be observed more clearly in Fig. 6. When $\delta \geq 0$ (i.e., frequency shift is larger than the orthogonal frequency), the BER is constant no matter what the value of $R$ is. When $\delta=-0.3$ (i.e., frequency shift is smaller than the orthogonal frequency), the performance is getting worse with larger $R$. Furthermore, comparison of the two delay times of $x$ and $y$ DoPs is shown in Fig. 7, where $\Delta_{1}=\tau_{x} / T$ and $\Delta_{2}=\tau_{y} / T$ are the normalized delay times with respect to the symbol duration. It shows that the performance is significantly affected by the values of parameter $\Delta_{1}$ but is not influenced by the values of $\Delta_{2}$. Therefore, the delay interference produced by the second-path signal of the other DoP can be completely ignored.

\section{Guard Band}

Because of the existence of guard band (as shown in Fig. 2), the spectrum of $S_{i x}(t)$ is not orthogonally allocated with $S_{(i-1) y}(t)$ (i.e., the normalized shifting frequency $\delta$ has to be larger than zero). Fig. 8 illustrates the relation between the BER and $\delta$. It shows that the BER is almost constant when $\delta$ is larger than zero. So the system performance does not change when the guard band exists.

\section{Effect of Doppler Shift}

The relation between the BER and the rolloff factor $\beta$ is shown in Fig. 9. The BER for $\Delta=0.2$ has a peak when $\beta$ is about 0.2. Similarly, for $\Delta=0.3$, the BER has a peak when $\beta$ is about 0.3 . It implies that the BER is degraded significantly when $\beta$ and $\Delta$ have about the same value. This phenomenon is caused by the Doppler shift. Generally speaking, a slight frequency shift caused by the channel impairments will dominate the system performance. When a slight frequency shift occurs, $m(t, x)$ has the form listed in Region I of Table I, and it has huge interference. Moreover, there is a second peak around $\beta=0.8$ in Fig. 9. In this case, $m(t, x)$ has the form listed in Region III of Table I, which will also result in large interference. Therefore, it is important to estimate the most probable $f_{D}$ and to choose an appropriate value of $\beta$ to avoid BER degradation.

\section{E. Performance Comparison}

Comparison with other systems is shown in Fig. 10. The performances of the proposed, conventional OFDM and dual-polarization canceler with bootstrap [12] systems are presented. The performance improvement of the proposed system over the 


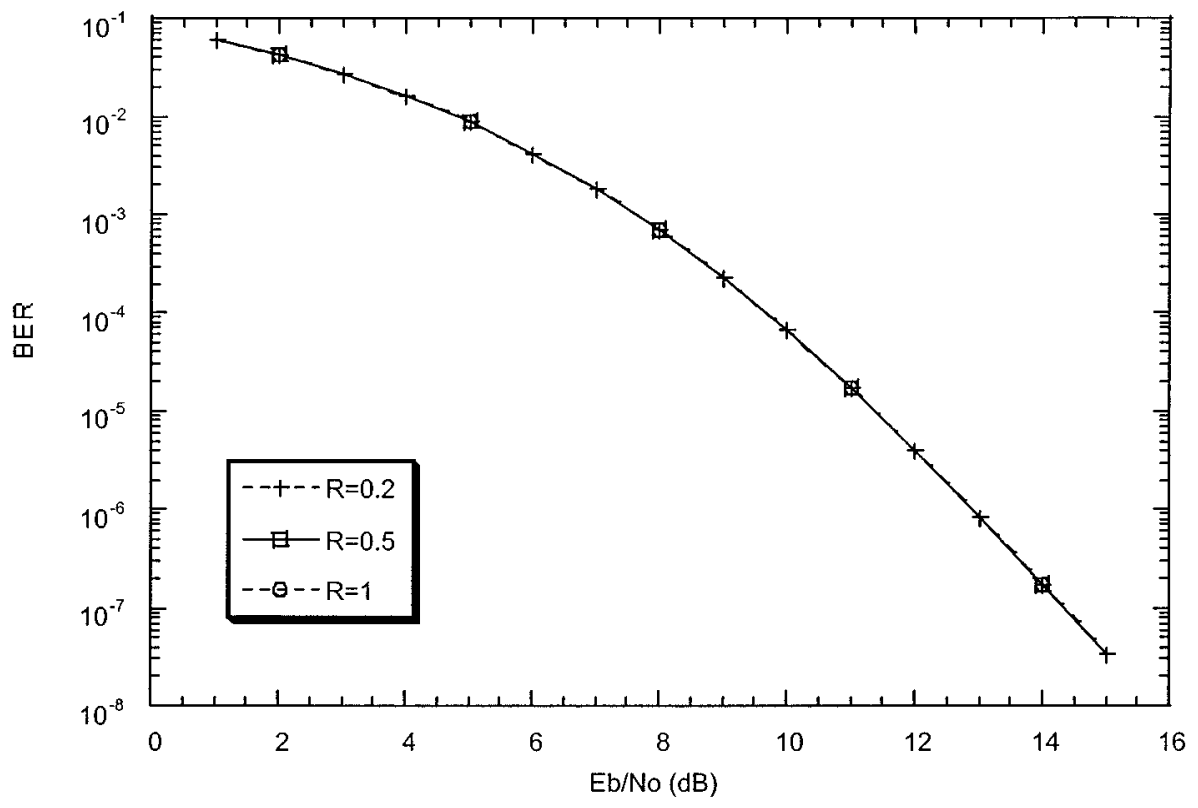

Fig. 5. Performance with various values of $R$ for $\Delta_{1}=0.2, \Delta_{2}=0.3, \delta=0.3, \gamma=0.2, \beta=0, \Delta=0.2$, and $\beta_{1}=0.95$.

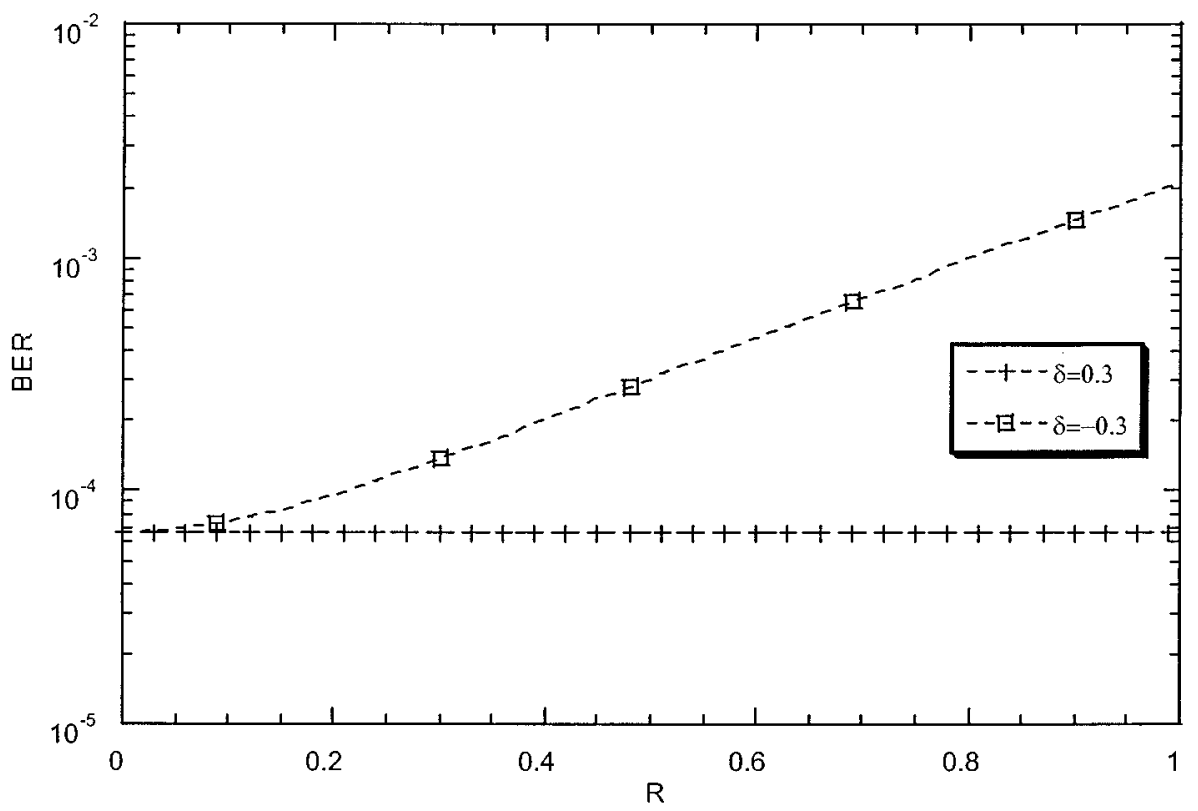

Fig. 6. Performance versus $R$ with various values of $\delta$ for $\Delta_{1}=0.2, \Delta_{2}=0.3, \gamma=0.2, \beta=0 \Delta=0.2, \beta_{1}=0.95$, and $E b / N 0=10(\mathrm{~dB})$.

dual-polarization canceler with bootstrap system is about three orders of magnitude at $E_{b} / N_{0}=14 \mathrm{~dB}$. The performance improvement over the conventional OFDM system is about 3.5 orders of magnitude at the same value of $E_{b} / N_{0}$. It is believed that the proposed system outperforms the other.

\section{CONCLUSIONS}

A new dual-polarization frequency reuse scheme with frequency allocation between $x$ and $y$ DoPs is presented without any complex compensation circuits. The system performance is improved significantly with little alteration of conventional dual-polarization systems. Unlike the conventional dual-polarization system, cross-polarization interference hardly influences the proposed system performance, and CPI can be totally ignored. The delay interference caused by the other DoP hardly impairs the desired symbol, and it simplifies the design for further improvement of the proposed system (e.g., equalization). Furthermore, unlike the OFDM system, multiple pilot tones can be added. The polarization recovery, carrier recovery, and channel estimation from the pilot tones are easier to implement. 


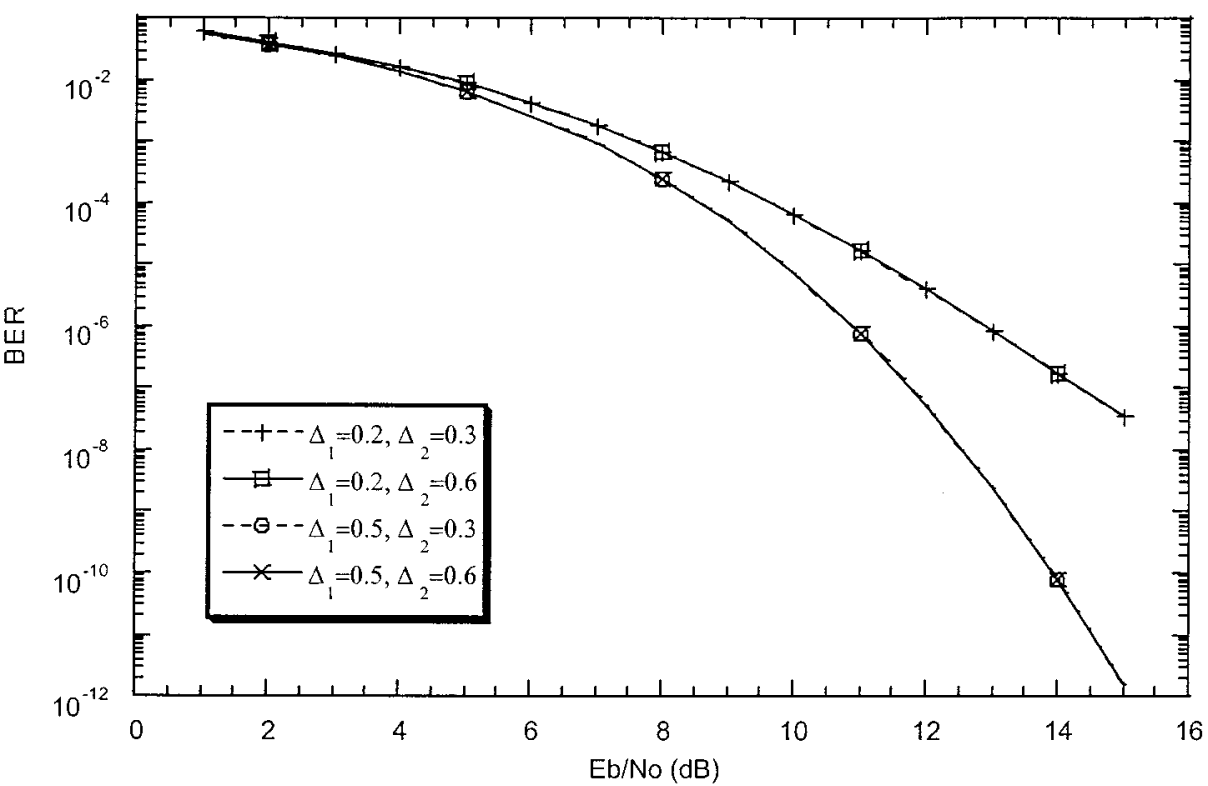

Fig. 7. Performance with various values of $\Delta_{1}$ and $\Delta_{2}$ for $\gamma=0.2, \delta=0.3, R=0.2, \beta=0, \Delta=0.2$, and $\beta_{1}=0.95$.

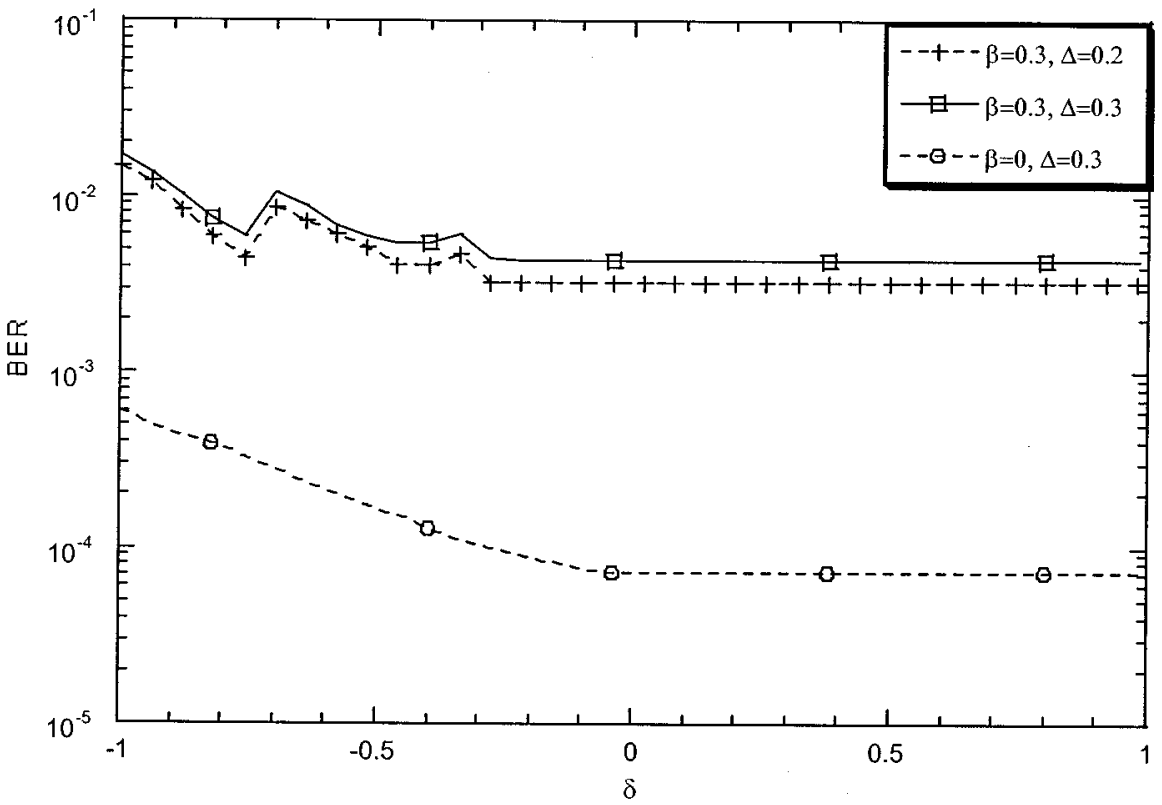

Fig. 8. Performance versus $\delta$ with various values of $\beta$ and $\Delta$ for $\Delta_{1}=0.2, \Delta_{2}=0.3, \gamma=0.2, R=0.2, \beta_{1}=0.95$, and $E b / N o=10(\mathrm{~dB})$.

\section{APPENDIX I}

Let $Q_{1}(f)$ be the Fourier transform of $q_{1}(t-k T, x)$ in (10) and $P(f)$ be the Fourier transform of the square-root raised cosine impulse response $p(t)$. $Q_{1}(f)$ is given by

$$
\begin{aligned}
Q_{1}(f)= & F\left\{q_{1}(t, x)\right\} \\
= & \frac{1}{2}\left[P\left(f-\frac{x}{T}\right) P(f) e^{-j 2 \pi(f-(x / T)) k T}\right. \\
& \left.\quad+P\left(f+\frac{x}{T}\right) P(f) e^{-j 2 \pi(f+(x / T)) k T}\right] \\
= & \frac{1}{2}\left[M^{\prime}(f) e^{j 2 \pi k x}+M^{\prime}\left(f+\frac{x}{T}\right) e^{-j 2 \pi k x}\right] e^{-j 2 \pi f k T}
\end{aligned}
$$

where

$$
M^{\prime}(f)=P\left(f-\frac{x}{T}\right) P(f)
$$

Taking the inverse Fourier transform, we obtain $q_{1}(t-k T, x)$ as

$$
\begin{aligned}
q_{1}(t-k T, x)= & F^{-1}\left\{Q_{1}(f)\right\} \\
= & \frac{1}{2}\left[m^{\prime}(t-k T, x) e^{j 2 \pi k x}\right. \\
& \left.\quad+m^{\prime}(t-k T, x) e^{-j(2 \pi x t / T)}\right] \\
=\frac{1}{2}[ & {\left[(t-k T, x) e^{j(\pi x(t+k T)) / T}\right.} \\
& \left.\quad+m(t-k T, x) e^{-j(\pi x(t+k T)) / T}\right] \\
= & \cos \left(\frac{\pi x}{T}(t+k T)\right) m(t-k T, x) .
\end{aligned}
$$

Equation (12) is proved. Equation (13) can be proved by the same fashion. In (A1-3), the functions $m^{\prime}(t, x)$ and $m(t, x)$ are 


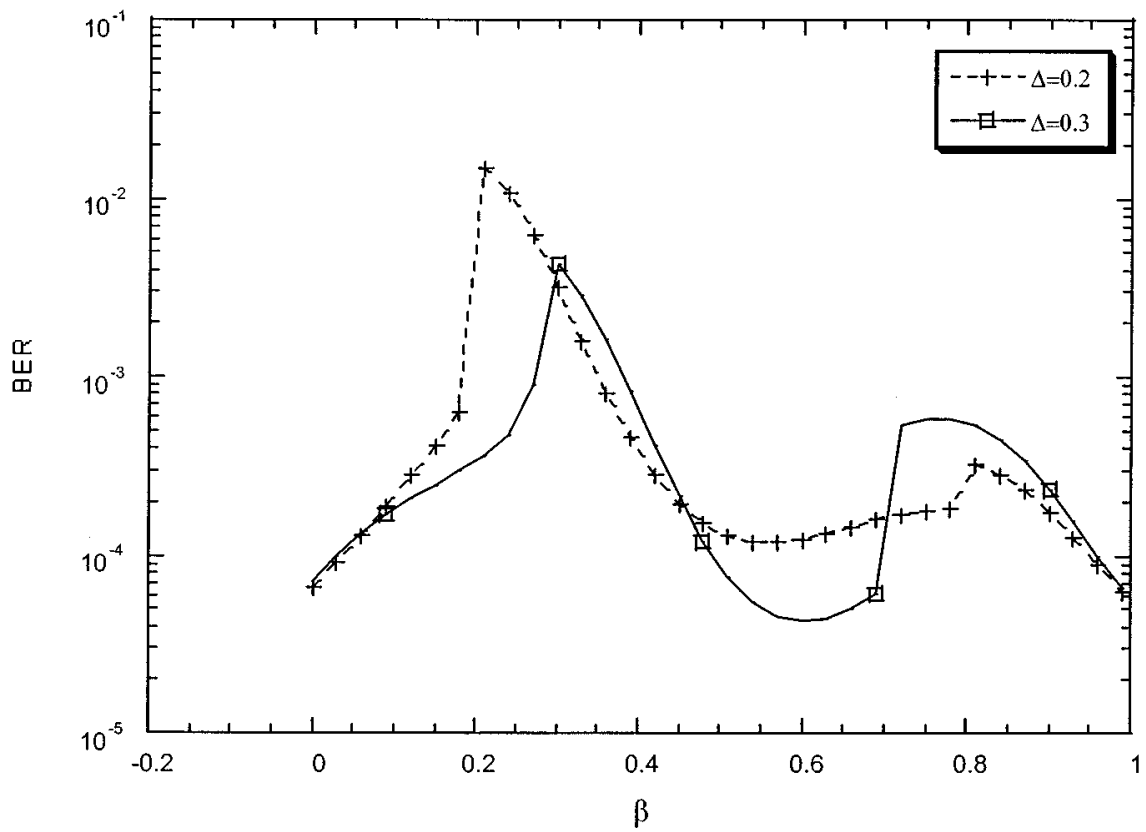

Fig. 9. Performance versus $\beta$ with various values of $\Delta$ for $\Delta_{1}=0.2, \Delta_{2}=0.3, \delta=0.3, \gamma=0.2, R=0.2, \beta_{1}=0.95$, and $E b / N o=10$ (dB).

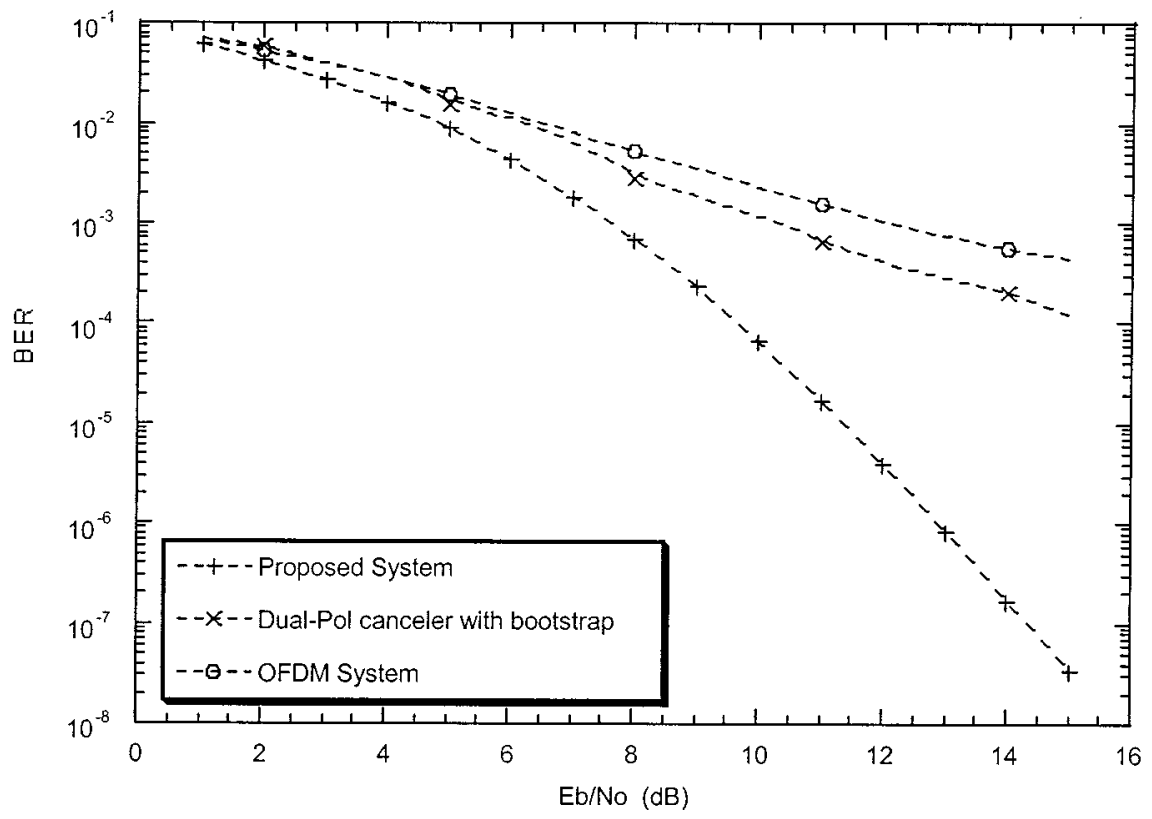

Fig. 10. Performance comparison with the dual-polarization cancellation system and the OFDM system.

given as

$$
m^{\prime}(t, x)=F^{-1}\{M(f)\}
$$

and

$$
\begin{aligned}
m(t, x) & =m^{\prime}(t, x) e^{-j(\pi x t / T)} \\
& =F^{-1}\left\{P\left(f-\frac{x}{2 T}\right) P\left(f+\frac{x}{2 T}\right)\right\} .
\end{aligned}
$$

The function of $m(t, x)$ can be obtained from (A1-5), where
$P(f)$ is given by

$$
P(f)= \begin{cases}\sqrt{T}, & 0 \leq|f| \leq \frac{1-\beta}{2 T} \\ \sqrt{T} \cos \left(\frac{\pi T|f|}{2 \beta}-\frac{\pi(1-\beta)}{4 \beta}\right), & \\ \frac{1-\beta}{2 T} \leq|f| \leq \frac{1+\beta}{2 T} & \\ 0, & \text { otherwise. }\end{cases}
$$

Based on the characteristic of $P(f)$, we have to divide our analysis according to $0 \leq \beta<0.5$ and $0.5 \leq \beta<1$, where $\beta$ is the roll off factor. The result of $m(t, x)$ is listed in Table I. 


\section{APPENDIX II}

The detailed derivation of (17) is shown in the following. First, let the phase terms $\alpha_{1}$ and $\alpha_{2}$ be constant. The conditional error probability is given by

$$
\begin{aligned}
P_{e}= & \operatorname{Pr}\left\{Z_{i x}<0 \mid a_{0}^{i}=1, \alpha_{1}, \alpha_{2}\right\} \\
= & \int_{0}^{\infty} \operatorname{Pr}\left\{N<-\left(1+X_{1}+A X_{2}\right) \mid \alpha_{1}, \alpha_{2}\right\} \\
& \cdot A e^{-\left(A^{2} / 2\right)} d A \\
= & \int_{0}^{\infty} Q\left(\sqrt{\frac{2}{N_{0}}}\left(1+X_{1}+A X_{2}\right)\right) A e^{-\left(A^{2} / 2\right)} d A \\
= & \int_{0}^{\infty} \int_{\sqrt{2 / N_{0}}\left(1+X_{1}+A X_{2}\right)}^{\infty} \frac{1}{\sqrt{2 \pi}} \\
& \cdot e^{-\left(n^{2} / 2\right)} A e^{-\left(A^{2} / 2\right)} d n d A
\end{aligned}
$$

where $Z_{i x}$ is shown in (14) and $N$ is a zero-mean AWGN with variance of $N_{0} / 2$. The bit energy $E_{b}$ has been normalized to unity. Let $z$ be the energy-to-noise ratio $1 / N_{0}$. After exchanging the inner integral and outer integral, we have $P_{e}$ as

$$
P_{e}= \begin{cases}\int_{\sqrt{2 z}\left(1+X_{1}\right)}^{\infty} \int_{0}^{\left((n / \sqrt{2 z})-1-X_{1}\right) / X_{2}} & \\ \frac{1}{\sqrt{2 \pi}} e^{-\left(n^{2} / 2\right)} A e^{-\left(A^{2} / 2\right)} d A d n, & X_{2}>0 \\ \left(\int_{-\infty}^{\sqrt{2 z}\left(1+X_{1}\right)} \int_{\left((n / \sqrt{2 z})-1-X_{1}\right) / X_{2}}^{\infty}\right. & \\ \left.+\int_{\sqrt{2 z}\left(1+X_{1}\right)}^{\infty} \int_{0}^{\infty}\right) & \\ \frac{1}{\sqrt{2 \pi}} e^{-\left(n^{2} / 2\right)} A e^{-\left(A^{2} / 2\right)} d A d n, & X_{2}<0 .\end{cases}
$$

After finishing integration, we get $P_{e}$ as

$$
\begin{aligned}
P_{e}= & \left\{\begin{array}{cc}
Q\left(\sqrt{2 z}\left(1+X_{1}\right)\right)-\sqrt{\frac{2 z X_{2}^{2}}{1+2 z X_{2}^{2}}} \\
\exp \left[-\frac{z\left(1+X_{1}\right)^{2}}{1+2 z X_{2}^{2}}\right] \\
Q\left(\frac{2 z X_{2}\left(1+X_{1}\right)}{\sqrt{1+2 z X_{2}^{2}}}\right), & X_{2}>0 \\
Q\left(\sqrt{2 z}\left(1+X_{1}\right)\right)+\sqrt{\frac{2 z X_{2}^{2}}{1+2 z X_{2}^{2}}} & X_{2}<0 \\
\exp \left[-\frac{z\left(1+X_{1}\right)^{2}}{1+2 z X_{2}^{2}}\right] & \left(-\frac{2 z X_{2}\left(1+X_{1}\right)}{\left.\sqrt{1+2 z X_{2}^{2}}\right),}\right. \\
= & Q\left(\sqrt{2 z}\left(1+X_{1}\right)\right)-x \sqrt{\frac{2 z}{1+2 z x^{2}}} \\
& \left.\cdot \exp \left[-\frac{\left.z\left(1+X_{1}\right)^{2}\right]}{1+2 z x^{2}}\right]\left(\frac{2 z x\left(1+X_{1}\right)}{\sqrt{1+2 z x^{2}}}\right)\right|_{x=X_{2}}
\end{array} .\right.
\end{aligned}
$$

So the overall bit error probability given in (17) can be obtained by

$$
P e_{d e p}=\left(\frac{1}{2 \pi}\right)^{2} \int_{-\pi}^{\pi} \int_{-\pi}^{\pi} P_{e}\left(\alpha_{1}, \alpha_{2}\right) d \alpha_{1} d \alpha_{2} .
$$

\section{APPENDIX III}

The derivation of (22) can be undertaken from (A2-3). Because there are two Rayleigh random variables $A_{x}$ and $A_{y}$ in Eq. (18), the integration has to be done one more time than that in Appendix II. Let $\alpha_{1}$ and $\alpha_{2}$ be constant. The conditional error probability is given by

$$
\begin{aligned}
P_{e}^{\prime}= & \operatorname{Pr}\left\{Z_{i x}<0 \mid a_{0}^{i}=1, \alpha_{1}, \alpha_{2}\right\} \\
= & \int_{0}^{\infty}\left[Q\left(\sqrt{2 z}\left(1+Y_{1}+A_{y} Y_{3}\right)\right)\right. \\
& -Y_{2} \sqrt{\frac{2 z}{1+2 z Y_{2}^{2}}} \exp \left[-\frac{z\left(1+Y_{1}+A_{y} Y_{3}\right)^{2}}{1+2 z Y_{2}^{2}}\right] \\
& \left.\cdot Q\left(\frac{2 z Y_{2}\left(1+Y_{1}+A_{y} Y_{3}\right)}{\sqrt{1+2 z Y_{2}^{2}}}\right)\right] A_{y} e^{-A_{y}^{2} / 2} d A_{y} . \\
= & P e_{d e p}\left(x=Y_{3}\right)-Y_{2} \sqrt{\frac{2 z}{1+2 z Y_{2}^{2}}} \\
& \cdot \int_{0}^{\infty} \exp \left[-\frac{z\left(1+Y_{1}+A_{y} Y_{3}\right)^{2}}{1+2 z Y_{2}^{2}}\right] \\
& \cdot Q\left(\frac{2 z Y_{2}\left(1+Y_{1}+A_{y} Y_{3}\right)}{\sqrt{1+2 z Y_{2}^{2}}}\right) A_{y} e^{-A_{y}^{2} / 2} d A_{y} .
\end{aligned}
$$

Let

$$
\begin{aligned}
P_{b}= & \int_{0}^{\infty} \exp \left[-\frac{z\left(1+Y_{1}+A_{y} Y_{3}\right)^{2}}{1+2 z Y_{2}^{2}}\right] \\
& \cdot Q\left(\frac{2 z Y_{2}\left(1+Y_{1}+A_{y} Y_{3}\right)}{\sqrt{1+2 z Y_{2}^{2}}}\right) A_{y} e^{-A_{y}^{2} / 2} d A_{y} .
\end{aligned}
$$

After exchanging the inner integral and outer integral of $P_{b}$, we have $P_{e}$ as

$$
P_{b}=\left\{\begin{array}{cc}
\frac{1}{\sqrt{2 \pi}} \int_{G\left(1+Y_{1}\right)}^{\infty} \int_{0}^{\left(\left(n-G\left(1+Y_{1}\right)\right) / G Y_{3}\right)} & \\
e^{C} A_{y} e^{-E\left(A_{y}+F\right)^{1}} e^{-n^{2} / 2} d A_{y} d n, & Y_{2} Y_{3}>0 \\
\frac{1}{\sqrt{2 \pi}}\left(\int_{-\infty}^{G\left(1+Y_{1}\right)} \int_{\left(\left(n-G\left(1+Y_{1}\right)\right) / G Y_{3}\right)}^{\infty}\right. & \\
\left.+\int_{G\left(1+Y_{1}\right)}^{\infty} \int_{0}^{\infty}\right) & \\
e^{C} A_{y} e^{-E\left(A_{y}+F\right)^{1}} e^{-n^{2} / 2} d A_{y} d n, & Y_{2} Y_{3}<0
\end{array}\right.
$$

where

$$
C=-\frac{z\left(1+Y_{1}\right)^{2}}{1+2 z Y_{2}^{2}+2 z Y_{3}^{2}}
$$




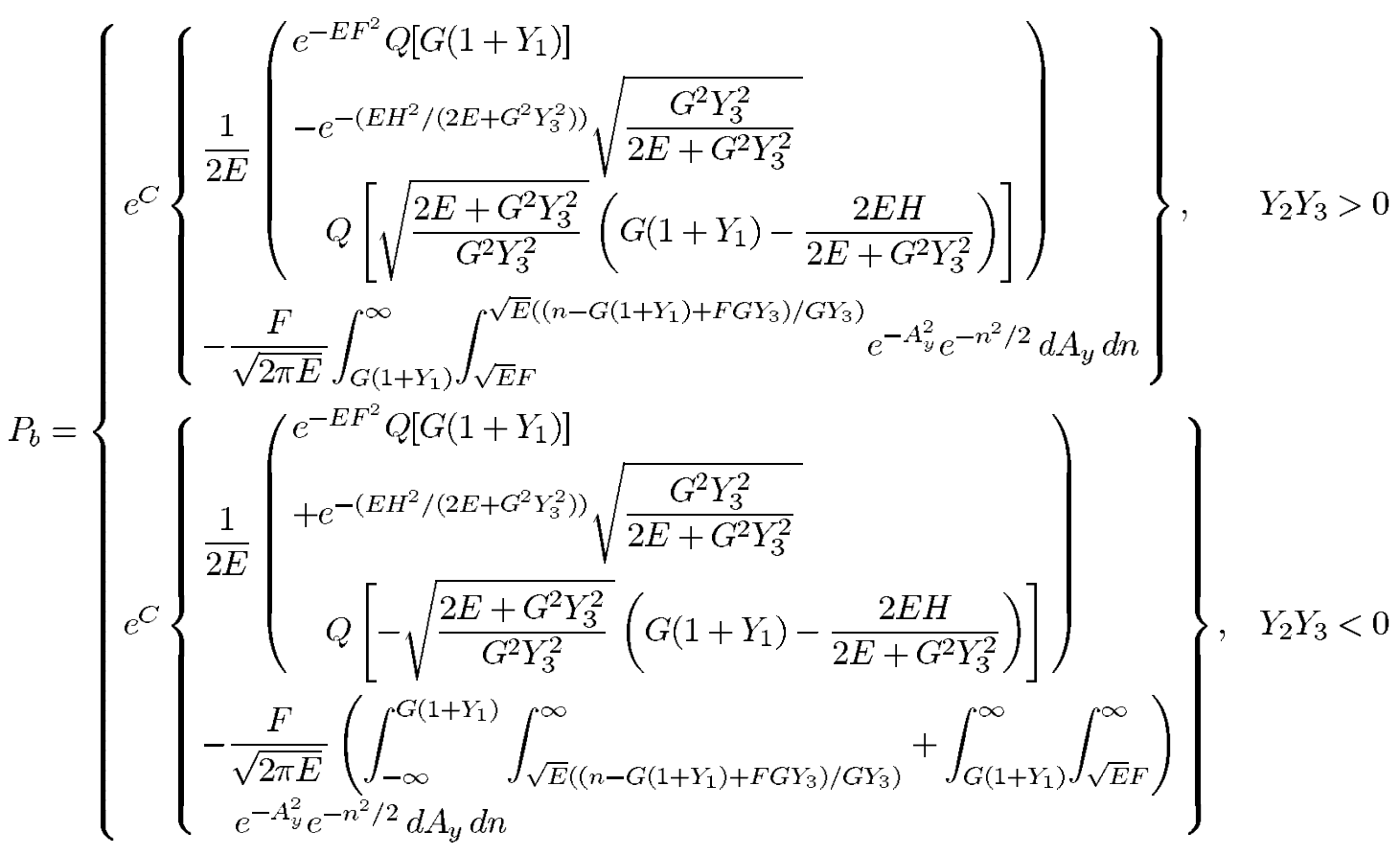

$$
\begin{aligned}
& =\frac{1+2 z Y_{2}^{2}}{1+2 z Y_{2}^{2}+2 z Y_{3}^{2}}\left[e^{-\left(\left(z\left(1+Y_{1}\right)^{2}\right) /\left(1+2 z Y_{2}^{2}\right)\right)} Q\left(\frac{2 z Y_{2}\left(1+Y_{1}\right)}{\sqrt{1+2 z Y_{2}^{2}}}\right)\right. \\
& \left.-3 \frac{2 z Y_{2} Y}{\sqrt{\left(1+2 z Y_{2}^{2}\right)\left(1+2 z Y_{3}^{2}\right)}} e^{-\left(\left(z\left(1+Y_{1}\right)^{2}\right) /\left(1+2 z Y_{3}^{2}\right)\right)} Q\left(\frac{2 z Y_{3}\left(1+Y_{1}\right)}{\sqrt{1+2 z Y_{3}^{2}}}\right)\right] \\
& -\frac{Y_{2} Y_{3}\left(1+Y_{1}\right)}{\sqrt{\pi}\left((2 z)^{-1}+Y_{2}^{2}+Y_{3}^{2}\right)^{3 / 2}} \exp \left[-\frac{z\left(1+Y_{1}\right)^{2}}{1+2 z Y_{2}^{2}+2 z Y_{3}^{2}}\right] \cdot \operatorname{Re} s
\end{aligned}
$$

$$
\begin{aligned}
& E=\frac{1+2 z Y_{2}^{2}+2 z Y_{3}^{2}}{2+4 z Y_{2}^{2}} \\
& F=\frac{2 z Y_{3}\left(1+Y_{1}\right)}{1+2 z Y_{2}^{2}+2 z Y_{3}^{2}} \\
& G=\frac{2 z Y_{2}}{\sqrt{1+2 z Y_{2}^{2}}} .
\end{aligned}
$$

After tedious manipulation, we obtain $P_{b}$ as shown in (A3-8) at the top of the page, where

$$
H=G\left(1+Y_{1}\right)-F G Y_{3}
$$

The overall error probability can be obtained by

$$
P_{e}=\left(\frac{1}{2 \pi}\right)^{2} \int_{-\pi}^{\pi} \int_{-\pi}^{\pi} P_{e}^{\prime}\left(\alpha_{1}, \alpha_{2}\right) d \alpha_{1} d \alpha_{2}
$$

Equations (22)-(24) are proved.

\section{ACKNOWLEDGMENT}

The authors wish to thank the reviewers for their valuable comments and suggestions.

\section{REFERENCES}

[1] W. Y. Zou and Y. Wu, "COFDM: An overview," IEEE Trans. Broadcasting, vol. 41, pp. 1-8, Mar. 1995.

[2] H. H. H'mimy, "Channel estimation based on coded pilot for OFDM," in Proc. IEEE Vehicular Technology Conf., vol. 3, 1997, pp. 1375-1379.

[3] D. T. Harvatin and R. E. Ziemer, "Orthogonal frequency division multiplexing performance in delay and doppler spread channels," in Proc. IEEE Vehicular Technology Conf., vol. 3, 1997, pp. 1644-1647.

[4] C. E. Hendrix, G. Kulon, C. S. Anderson, and M. A. Heinze, "Multigigabit transmission through rain in a dual polarization frequency reuse system: An experimental study," IEEE Trans. Commun., vol. 41, pp. 1830-1837, Dec. 1993.

[5] L. Ordano and F. Tallone, "Dual polarized propagation channel: Theoretical model and experimental results," in Proc. 10th Int. Conf. Antennas and Propagation, vol. 2, Apr. 1997, pp. 363-366.

[6] T. S. Chu, "Restoring the orthogonality of two polarizations in radio communication systems I," Bell Syst. Tech. J., vol. 50, no. 9, pp. 3063-3069, Nov. 1971.

[7] - "Restoring the orthogonality of two polarizations in radio communication systems II," Bell Syst. Tech. J., vol. 52, no. 3, pp. 3063-3069, Mar. 1973.

[8] B. Lankl, J. A. Nossek, and G. Sebald, "Cross-polarization interference in the presence of delay effects," in Proc. IEEE Int. Conf. Communications, vol. 3, 1988, pp. 1355-1361.

[9] M. Kavehrad and J. Salz, "Cross-polarization cancellation and equalization in digital transmission over dually polarized multipath fading channels," Bell Syst. Tech. J., vol. 64, no. 10, pp. 2211-2245, Dec. 1985.

[10] Parsons, The Mobile Radio Propagation Channel. New York, NY: Wiley.

[11] J. G. Proakis, Digital Communications, 2nd ed. New York: McGrawHill, May 1993/1989. 
[12] H. Ge, Y. Bar-Ness, and M. Visser, "Combined adaptive interference cancellation and bootstrap separation of dual polarized signals," in Conf. Rec. 30th Asilomar Conf. Signals, Systems and Computers, vol. 1, 1996, pp. 694-698.

[13] G. Santella, "Bit error rate performances of M-QAM orthogonal multicarrier modulation in presence of time-selective multipath fading," in Proc. IEEE ICC'95, Seattle, WA, 1995, pp. 1683-1688.

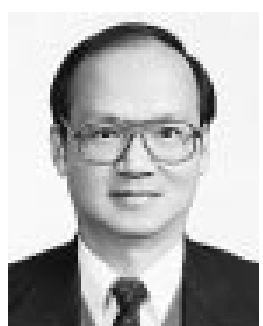

Jingshown Wu (S'73-M'79-SM'99) received the

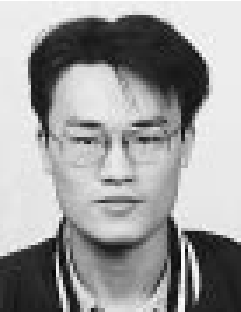
B.S. and M.S. degrees in electrical engineering from National Taiwan University, Taipei, Taiwan, R.O.C., in 1970 and 1972, respectively, and the Ph.D. degree from Cornell University, Ithaca, NY, in 1978.

He joined Bell Laboratories in 1978, where he worked on digital network standards and performance and optical fiber communication systems. In 1984, he joined the Department of Electrical Engineering, National Taiwan University, as a Professor, and was Chairman of the department from 1987 to 1989. He was also Director of the university's Communication Research Center, College of Engineering, from 1992 to 1995. From 1995 to 1998, he was Director of the Division of Engineering and Applied Science, National Science Council, R.O.C., on leave from the university. Since 1999, he has become Chairman of the Commission on Research and Development and Director of the Center for Sponsor Programs of National Taiwan University. $\mathrm{He}$ is interested in optical fiber communications, wireless communication, and computer communication networks.

Prof. Wu is a Life Member of the Chinese Institute of Engineers, the Optical Society of China, and the Institute of Chinese Electrical Engineers. He is Chairman of the IEEE Taipei Section.
Meng-Che Wu was born in Taipei, Taiwan, R.O.C., in 1975. He received the B.S. and M.S. degrees in electrical engineering from the National Taiwan University, Taipei, in 1997 and 1999, respectively.

$\mathrm{He}$ joined the Wireless Communication Technology Lab, Chunghwa Telecom Laboratories, Taoyuan, Taiwan, in 1999. His research interests include modulation techniques, coding, mobile communication, spread-spectrum communication, and protocol analysis. 